\title{
Enfermedad ateroembólica y hemorragia pulmonar
}

\author{
F. J. DE LA PRADA ÁLVAREZ, C. BLANCO HUELGA ${ }^{1}$, A. M. PRADOS \\ GALLARDO $^{2}$, M. URIOL RIVERA, E. ANTÓN VALENTI ${ }^{3}$, A. MOREY MOLINA \\ Servicios de Nefrología, ${ }^{1}$ Cuidados Intensivos y ${ }^{3}$ Anatomía Patológica. Hospital \\ Universitario Son Dureta. Palma de Mallorca. ${ }^{2}$ Servicio de Medicina Interna. Hospital \\ Virgen Macarena. Sevilla
}

ATHEROEMBOLIC DISEASE AND PULMONARY HAEMORRHAGE

\begin{abstract}
RESUMEN
El síndrome de embolismo de colesterol se produce por la liberación de cristales de colesterol de placas ateroscleróticas ulceradas de grandes arterias. Puede afectar múltiples órganos simulando enfermedades sistémicas como las vasculítis, y debe ser incluido en el diagnóstico diferencial de los síndromes neumo-renales.

El diagnóstico de embolismo de colesterol debería contemplarse en pacientes de edad, con enfermedad aterosclerótica previa, que desarrollan fallo renal o deterioro de una insuficiencia renal previa, y en los que aparecen hallazgos clínicos sugestivos de embolismos de colesterol periféricos, asociado a factores precipitantes, sin necesidad de demostración histológica.

Este síndrome se ha asociado tradicionalmente a una alta morbi-mortalidad, aunque en recientes series se sugiere que un manejo agresivo con medidas de soporte, evitando factores precipitantes y el uso de corticoides a bajas dosis pueden proporcionar una mejoría en su pronóstico.
\end{abstract}

PALABRAS CLAVE: Embolización de cristales de colesterol. Atheroembolic disease.

\begin{abstract}
The cholesterol embolism syndrome is a multisystemic disease resulting from cholesterol crystal emboli deriving from ulcerous atherosclerotic plaques of the aorta or large arteries. Cholesterol crystal embolization can affect multiple organ, including the skin, kidney, brain, eyes, gastrointestinal tract and extremities, and mimic other systemic diseases like vasculitis. Cholesterol crystal embolization of lungs has been described and should be included in the differential diagnostic of pulmonaryrenal syndromes.

The diagnosis of cholesterol embolism should be considered in elderly patients with pre-existing atherosclerotic disease who develop renal failure and clinical features of peripheral cholesterol crystal embolization in association with precipitanting event, without the need for histological demostration of cholesterol clefts.

This syndrome is associated with high morbidity and mortality but recents reports suggest that an aggresive therapeutic management with patient-tailored supportive measures, avoids precipitating factors, and the use of corticosteroids may be associated with a favorable clinical outcome.
\end{abstract}

KEY WORDS: Cholestherol crystal embolization. Atheroembolic disease.

De la Prada Álvarez FJ, Blanco Huelga C, Prados Gallardo AM, Uriol Rivera M, Antón Valenti E, Morey Molina A. Enfermedad ateroembólica y hemorragia pulmonar. An Med Interna (Madrid) 2004; 21: 231-234.

\section{INTRODUCCIÓN}

El síndrome de embolismo de colesterol se produce por la liberación de cristales de colesterol de placas ateroscleróticas ulceradas de grandes arterias. Puede afectar múltiples órganos simulando enfermedades sistémicas como las vasculítis, y debe ser incluido en el diagnóstico diferencial de los síndromes neumo-renales.

El diagnóstico de embolismo de colesterol debería contemplarse en pacientes de edad, con enfermedad ateroscleróti- ca previa, que desarrollan fallo renal o deterioro de una insuficiencia renal previa, y en los que aparecen hallazgos clínicos sugestivos de embolismos de colesterol periféricos, asociado a factores precipitantes, sin necesidad de demostración histológica.

Este síndrome se ha asociado tradicionalmente a una alta morbi-mortalidad, aunque en recientes series se sugiere que un manejo agresivo con medidas de soporte, evitando factores precipitantes y el uso de corticoides a bajas dosis pueden proporcionar una mejoría en su pronóstico. 


\section{CASO APORTADO}

Paciente de 66 años de edad con antecedentes de diabetes mellitus de un año de evolución conocida, fumador de 20 cigarrillos diarios e hipertensión arterial de 40 años de evolución. En noviembre de 1999 sufre episodio de pérdida brusca de agudeza visual en ojo derecho, con recuperación completa, diagnosticándose de embolia retiniana. Se realiza arteriografía carotídea, detectándose estenosis del $70 \%$ en carótida derecha y del $40 \%$ en la izquierda, proponiéndose endarterectomía de forma reglada; antes del procedimiento radiológico presentaba una cifra de creatinina plasmática de 1,6 mg/dl.

Un mes después ingresa para la realización de la cirugía, detectándose valores de creatinina de $17 \mathrm{mg} / \mathrm{dl}$, urea $317 \mathrm{mg} / \mathrm{l}$, potasio 6,1 mEq/1, pH 7,23, HCO3 17,2 y EB -10,6, refiriendo en el último mes clínica de claudicación intermitente, náuseas, vómitos, dolor abdominal y aparición de lesiones violáceas en piernas, compatibles con livedo reticularis, extendiéndose hasta dedos de los pies.

No se detectó eosinofília. Las inmunoglobulinas, complemento, ANA, ANCA, anti-DNA, anti-MBG, crioglobulinas, factor reumatoideo, serologias de hepatitis B y C, VIH y serología de neumonía atípica fueron negativos. La ecografía renal mostraba riñón derecho disminuido de tamaño con mala diferenciación corticomedular y aumento de la ecogenicidad; el izquierdo era ligeramente hipoplásico con discreto grado de atrofia cortical; no habia signos obstructivos. En el fondo de ojo se detectaron cristales de colesterol en las arteriolas. En el ecocardiograma transesofágico se objetivó ateromatosis severa en cayado y aorta torácica descendente, engrosamiento intimal y múltiples lesiones ulceradas. El eco-doppler de troncos viscerales demostró aorta infrarrenal ateromatosa y aneurismática.

Con el diagnóstico clínico de enfermedad ateroembólica renal se inicia tratamiento sustitutivo mediante hemodiálisis sin heparina, a través de un catéter tunelizado. Durante este ingreso el paciente continuó presentando episodios autolimitados de dolor abdominal epigástrico y las lesiones de las extremidades inferiores no mostraron mejoría, pero tampoco evolucionaron hacia lesiones necróticas. El estado general del paciente era aceptable y pudo ser dado de alta a su domicilio.

Tras un mes de tratamiento sustitutivo renal y sin mejoría analítica de la función renal, el paciente comienza a presentar hemoptisis de repetición y desarrollo de un cuadro de púrpura en MMII, precisando ingreso en la Unidad de Cuidados Intensivos por deterioro de la función pulmonar, con sospecha clínica de hemorragia pulmonar. No se encontraron lesiones en mucosa orofaríngea. El estudio de coagulación fue normal. La radiología de tórax mostraba infiltrados alveolares bilaterales con predominio en base pulmonar izquierda. El paciente precisó ventilación mecánica por insuficiencia respiratoria grave, tras lo cual se objetivó una remisión de la hemoptisis. Ante la posibilidad clínica de una vasculítis, se inició tratamiento con 5 bolus de esteroides, sin observarse mejoría de la situación clínica, de la radiología de tórax, ni de la gasométria. Se colocó catéter de SwanGanz en arteria pulmonar con ausencia de datos sugestivos de tromboembolismo pulmonar y edema agudo de pulmón. Se practicó fibrobroncoscopia que mostró ausencia de hemorragia y presencia de secreciones abundantes, sobre todo en LII. El estudio citológico del lavado alveolar mostró frotis de fondo hemático con predominio de macrófagos y neutrofilos sugestivo de inflamación aguda. La tomografía torácica mostró ocupación alveolar bilateral con atelectasia en lóbulo inferior izquierdo, enfisema pulmonar, ateromatosis aórtica y ausencia de adenopatias. Se realizó biopsia de las lesiones cutáneas de miembros inferiores, encontrando infiltración grasa con ausencia de signos de vasculitis. No se realizaron biopsia pulmonar ni renal porque la situación clínica del paciente no lo permitió. Finalmente presentó episodio de hemorragia digestiva alta con gran repercusión en la situación clínica general, siendo este el motivo final del fallecimiento.

En la necropsia se detectaron lesiones de ateroembolismo de colesterol a nivel pericárdico, espacios porta hepáticos, arterias de mediano y pequeño calibre pancreáticas, bazo, vasos de submucosa y mesenterio de tubo digestivo, vasos pericapsulares suprarrenales, arterias de pequeño y mediano calibre renales (Figs. 1), vasos intersticiales de testículos, próstata, meninges y vasos intraparenquimatosos cerebrales. Además a nivel pancreático se observaba necrosis del parénquima con áreas de esteatonecrosis, lesiones isquémicas y

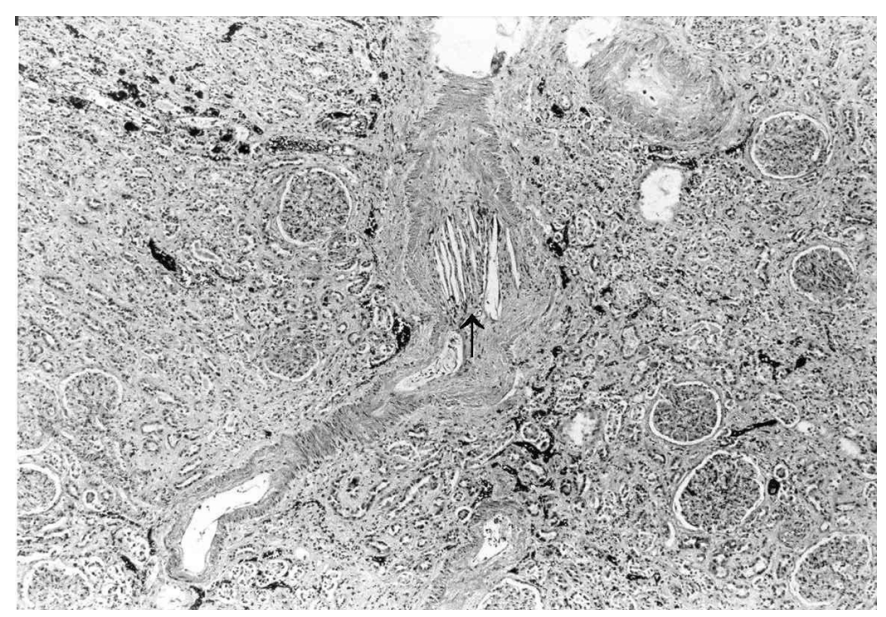

Fig. 1. Imagen histológica de ateroembolismo renal. Los cristales de colesterol aparecen como espacios claros biconcavos (flechas) en la luz de las arteriolas renales (hematoxilina-eosina $10 \times 10$ ).

necróticas en intestino grueso, junto con necrosis centrolobulillar hepática.

A nivel pulmonar no se objetivaron trombos ateroembólicos, pero sí infartos pulmonares focales, que explicaban la hemoptisis, y espacios alveolares ocupados por edema, histiocitos, neutrófilos, linfocitos y hemosiderófagos.

En la piel, las lesiones compatibles con lívedo reticularis y acrocianosis, demostraron áreas hemorrágicas a nivel de dermis papilar con marcada extravasación hemática, y necrosis coagulativa focal en epidermis sin signos de vasculitis ni detección de trombos ateroembólicos.

\section{DISCUSIÓN}

El síndrome de embolismo de colesterol es una causa reconocida de insuficiencia renal o de deterioro agudo de insuficiencia renal previa, y se presenta cada vez con mayor incidencia debido a la generalización de la cirugía cardiovascular, de los procedimientos radiológicos invasivos y del tratamiento anticoagulante en pacientes con patología aterosclerótica previa. Fue inicialmente descrito por Panum en 1862(1), y posteriormente etiquetado como entidad clínica por Flory en 1945, al detectar cristales de colesterol ocluyendo arterias de diámetro comprendido entre 55 y 900 micras en autopsias de pacientes con severa aterosclerosis y placas ateroscleroticas ulceradas(2). Posteriormente se produce una respuesta inflamatoria, proliferación endotelial, fibrosis tisular y oclusión de la luz vascular

Ocurre generalmente en pacientes mayores de 60 años, generalmente varones, hipertensos y fumadores, con lesiones ateroescleróticas difusas y antecedentes de enfermedad vascular cerebral, coronaria, aórtica, renal y/o periférica (3-6).

Suele estar precedido entre unas tres a ocho semanas antes 
por factores precipitantes tales como: a) procedimientos radiológicos invasivos (85\%) como aortografía, angiografía coronaria (el procedimiento mas comúnmente involucrado), cateterismo cardiaco, angioplastias sobre arterias coronarias, renales $\mathrm{u}$ otros sistemas arteriales $(5,6)$; b) tratamientos anticoagulantes $(76 \%)$ con dicumarínicos $(5,6)$, heparina sódica $(5,6)$, heparinas de bajo peso molecular $(5,7)$, o agentes trombolíticos (6); c) cirugía cardiaca o vascular (36\%) como resección de aneurisma aórtico abdominal, bypass aortoilíaco o aortofemoral, endarterectomía carotídea, cirugía cardiaca o injerto de arteria renal $(5,6)$; y d) y otras situaciones como traumatismos abdominales (8), con el uso de balón de contrapulsación aórtica (9), y en pocos casos están ausentes los factores precipitantes $(5,6)$.

Su presentación clínica varía desde pacientes asintomáticos, detectándose los cristales en estudios necropsicos, hasta la aparición de una enfermedad sistémica multiorgánica con fallo renal, fallo cardíaco, isquemia gastrointestinal, pancreatitis, hepatitis, encefalopatía, isquemia muscular y/o necrosis cutánea (3-6). La afectación de los diferentes órganos depende de la localización de las placas ateroscleróticas. Así la embolización desde placas carotídeas afectará a la circulación retiniana o cerebral, desde aorta torácica o abdominal al sistema nervioso central, órganos viscerales y extremidades, y desde aorta abdominal infrarrenal exclusivamente a las extremidades inferiores (6). Aunque puede afectar cualquier órgano de forma silente, los más frecuentemente involucrados son la piel (35-95\%) con la aparición de livedo reticularis, cianosis digital en miembros inferiores con pulsos periféricos conservados (síndrome del pie azul), nódulos, púrpura, petequias, gangrena o ulceras $(3-6,10)$; riñón cuya afectación ocurre en mas de un $50 \%$ de los casos debido a su alto flujo sanguíneo y a la proximidad de los riñones a la aorta abdominal, con la aparición de insuficiencia renal aguda (35\%), subaguda $(56 \%)$ o crónica (9\%), hipertensión arterial, infarto renal (3-6), microhematuria y/o proteinuria incluso de rango nefrótico $(5,11)$; tracto gastrointestinal en un 20-50\% según las series, generalmente, implicando un mal pronostico vital, porque implica afectación de sistema arterial mesenterico; las manifestaciones intestinales suelen tener una incidencia de 10$33 \%$, pudiendo aparecer anorexia, nauseas, vómitos, dolor abdominal postpandrial, diarrea, malabsorción, hemorragia gastrointestinal por ulceras o infartos de las mucosas, rectorragias, obstrucción intestinal por fibrosis, infarto o perforación intestinal, seudopolipos intestinales, pancreatitis, hepatitis, colecistitis necrotizantes acalculosas, infartos esplénicos o lesiones angiodisplásicas $(5,6,12-16)$; y sistema nervioso central que se manifiesta clínicamente en un 4-23\% de los pacientes, en forma de accidentes isquémicos transitorios, infartos cerebrales, amaurosis fúgax, parálisis, estados confusionales y deterioro neurológico progresivo $(3,6)$. La afectación retiniana ocurre en un 10-25\% de los pacientes, pudiéndose descubrir los émbolos de colesterol en las arterias retinianas en el estudio del fondo de ojo (placas de Hollenhorst) (17).

La afectación pulmonar se presenta infrecuentemente (1821) describiéndose la aparición de hemoptísis y distress respiratorio, lo que obliga al diagnóstico diferencial con otros síndromes neumo-renales. Se han conseguido objetivar cristales de colesterol en arterias bronquiales y pulmonares $(18,19)$, que en una ocasión se ha relacionado con la existencia de una fístula arteriovenosa que permite el paso de émbolos de colesterol hacia el pulmón (20).
La presentación clínica puede ser confundida fácilmente con multitud de procesos, y entre otros, con cuadros de vasculítis sistémicas, ya que puede manifestarse inespecificamente con fiebre, perdida de peso, mialgias o cefalea (6,22-24).

Los hallazgos de laboratorio son inespecificos apareciendo alterados los marcadores de inflamación (Velocidad de Sedimentación Glomerular, Proteina C Reactiva, Fibrinogeno) y marcadores que expresan la afectación de los diferentes órganos (hiperamilasemia en la afectación pancreática, elevación de la creatinfosfoquinasa en la afectación muscular, elevación de urea y creatinina en la afectación renal y elevación de transaminasas, fosfatasa alcalina y lacticodeshidrogenasa en la afectación hepática). Suele detectarse eosinofilia transitoria en la fase activa de la enfermedad en un 15-70\% de los casos, aunque es un hallazgo poco específico, e hipocomplementemia leve y transitoria (25), aunque esto último no se ha confirmado en series posteriores $(5,6)$.

Tradicionalmente se ha requerido el diagnóstico anatomopatológico, mediante biopsia cutánea (si hay afectación cutánea la biopsia suele ser diagnóstica en un $90 \%$ de casos (3), aunque varia entre un $50 \%$ y $80 \%$ de los casos (6)), gastrointestinal, muscular, renal o de medula ósea(6). No obstante el embolismo de colesterol puede ser diagnosticado clínicamente cuando se desarrolla fallo renal después de concurrir factores predisponentes conocidos para esta enfermedad, junto con manifestaciones sistémicas de embolización ateromatosa y alteraciones de laboratorio como las anteriormente descritas $(5,6)$. Así evitaríamos realizar procedimientos de riesgo, como es la biopsia renal, en pacientes críticos.

En nuestro paciente se realizó el diagnóstico clínico de Ateroembolia de colesterol en función de los factores de riesgo que presentaba, de las manifestaciones clínicas, junto con la detección de cristales de colesterol en el fondo de ojo y la presencia de ateromatosis aórtica severa con múltiples placas ulceradas. No pudo realizarse diagnóstico anatomopatológico, previo al exitus, porque no se detectaron cristales de colesterol a nivel cutáneo en la biopsia realizada (probablemente por la naturaleza parcheada de la lesión), y se desestimó la biopsia renal debido al estado clínico del paciente. En cuanto a las manifestaciones pulmonares, nuestro paciente no presentaba fístula arteriovenosa conocida, y a pesar de su búsqueda exhaustiva, no se pudieron demostrar émbolos de colesterol a nivel pulmonar en la necropsia. La necropsia demostró la afectación multisistemica que ocurre en esta enfermedad.

Las principales causas de mortalidad en este sindrome suelen ser los brotes recurrentes de embolismo de colesterol, el fallo cardíaco y la caquexia (5). Aunque tradicionalmente la mortalidad varía entre un $60-80 \%$ (4), y se ha considerado como una entidad básicamente intratable, el tratamiento agresivo de mantenimiento con la retirada de los factores pecipitantes (anticoagulación, manipulación aórtica, tóxicos renales, etc...), el control de la hipertensión, de la hiperlipidemia, del fallo cardiaco, el aporte nutricional y el tratamiento sustitutivo de la función renal, ha permitido alcanzar buenas tasas de supervivencia en series modernas (70-80\% en el primer año) $(5,6)$. Hay datos aislados del posible beneficio del tratamiento hipolipemiante como estabilizador de la placa aterosclerótica, sobre la evolución de la enfermedad $(5,6)$.

En un $40-60 \%$ de pacientes la insuficiencia renal suele ser definitiva y requiere de tratamiento sustitutivo permanente de la función renal $(5,6)$, aunque también se han comunicado casos de recuperación tras un periodo de trata- 
miento dialítico (4-6). En estos pacientes se ha sugerido que la técnica de tratamiento sustitutivo renal de elección debería ser la diálisis peritoneal, que evita el uso de anticoagulantes que impiden la estabilización de la placa ulcerada, y evita el estrés directo sobre el sistema cardiovascular que produce la hemodiálisis. Además la realización de ésta última puede hacer reaparecer los fenómenos ateroembólicos (26). La recuperación de la función renal podría ser más probable con el uso de la diálisis peritoneal, pero desgraciadamente las condiciones de estos pacientes, con malnutrición, isquemia intestinal o cirugía abdominal previa, no permiten habitualmente el uso de la misma. Si se usa hemodialisis, deberia realizarse con las minimas dosis posibles de heparina o sin ella.

Recientemente se ha enfatizado el uso precoz de bajas dosis de corticosteroides (0,3-0,5 mg/kg peso), por su acción antiinflamatoria y estabilizadora de la placa aterosclerótica
(5,21,27-30), en pacientes con evidencia de inflamación como nuevos episodios de ateroembolismo, eosinofilia recurrente o deficiente estado general, sin incremento de las posibles complicaciones por su uso, y con mejoria del dolor abdominal, del dolor en las lesiones digitales y en la anorexia. Además hay diferencia en el pronóstico de la función renal según se produzca el embolismo de colesterol sobre riñones nativos o sobre injertos renales, y los corticosteroides utilizados en estos últimos podría influir en su mejor evolución (31). No obstante, en otras series, el tratamiento agresivo de mantenimiento, sin el uso de esteroides, también ha permitido una buena supervivencia $(5,6)$.

Es preciso, dada la morbimortalidad asociada, realizar prevención de este sindrome restringiendo las indicaciones de procedimientos radiológicos invasivos o con contraste, de procedimientos quirurgicos vasculares y de tratamientos anticoagulantes en pacientes ateroscleróticos severos.

\section{Bibliografía}

1. Panum PL. Experimentelle Beiträge zur Lehre von der Embolie. Virchows Arch 1862; 25: 308-310.

2. Flory CM: Arterial occlusions produced by emboli from eroded atheromatous plaques. Am J Pathol 1945; 21: 549-565.

3. Fine MJ, Kapoor W, Falanga V. Cholesterol crystal embolization: a review of 221 cases in the English literature. Angiology 1987; 38: 769784.

4. Thadhani R, Camargo C, Xavier R, Fang L, Bazari H: Atheroembolic renal failure after invasive procedures: Natural history based on 52 histopathologically proven cases. Medicine (Baltimore) 1995; 74: 350-358.

5. Belefant X, Meyrier A, Jacquot C. Supportive Treatment Improves Survival in Multivisceral Cholesterol Crystal Embolism. Am J Kidney Dis 1999; 33: 840-850.

6. Scolari F, Tardanico R, Zani R, Pola A, Viola BF, Movilli E, Maiorca R. Colesterol Cristal Embolism: A Recognizable cause of renal Disease. Am J Kidney Dis 2000; 6: 1089-1109.

7. Belenfant X, d`Auzac C, Bariety J, Jacquot C. Cholesterol crystal embolism during treatment with low-molecular-weight heparin. Presse Med 1997; 26: 1236-7.

8. Shindo S, Okamoto H, Nagai M, et al . Acute ischemia of the lower legs from blunt abdominal trauma: an unusual case of atheroembolism-case report. J Trauma 1994; 36: 451-453.

9. Dahlberg PJ, Frecentese DF, Cogbill TH: Cholesterol embolism: Experience with 22 histologically proven cases. Surgery 1989; 105: 737-746.

10. Falanga V, Fine MJ, Kapoor WN. The cutaneous manifestations of cholesterol crystal embolization. Arch Dermatol 1986; 122: 1194-98.

11. Haqqie SS, Urizar RE, Singh J. Nephrotic-Range Proteinuria in Renal Atheroembolic Disease: Report of four cases. Am J Kidney Dis 1996; 28: 493-501.

12. Jiménez-Heffernan JA, Martinez García M, Burgos E. Small bowel perforation due to cholesterol atheromatous. Dig Dis Sci 1995; 40: 481184.

13. Mollenaar W, Lamers CB: Cholesterol crystal embolization and the disgestive system. Scand J Gastroent 1991; 188: 69-72.

14. Socindki MA, Frankel JP, Morow PL, Krawitt EL: Painless diarrhea secondary to intestinal ischemia. Diagnosis of atheromatous emboli by jejunal biopsy. Dig Dis Sci 1984; 51: 674-677.

15. Case records of the Massachussets General Hospital (Case 24-1998). N Engl J Med 1998; 339: 329-337.
16. Balian A, Gaudric M, Guimbaud R, Sogni P, Couterier D, Chaussade S. Cholesterol crystal embolization in the digestive tract. Gastroenterol Clin Biol 1998; 22: 290-297.

17. Gittinger JW, Kershaw GR. Retinal Cholesterol Emboli in the Diagnosis of Renal Atheroembolism. Arch Intern Med 1998; 11: 1265-7.

18. Sabatine MS, Oelberg DA, Mark EJ, Kanarek D. Pulmonary cholesterol crystal embolization. Chest 1997, 112: 1687-92.

19. Case records of the Massachussets General Hospital (Case 11-1996). N Engl J Med 1996; 334: 973-979.

20. Weigent CE. Pulmonary atheroembolism complicating repair of an atherosclerotic abdominal aneurysm. Minn Med 1978; 61: 15-16.

21. Vacher-Coponat H, Pache X, Dussol B, Berland Y. Pulmonary-renal syndrome responding to corticosteroids: consider cholesterol embolization. Nephrol Dial Transplant 1997; 12: 1977-1979.

22. Young DK, Burton MF, Herman JH. Multiple cholesterol emboli syndrome simulating necrotizing vasculitis. J Rheumatol 1986; 13: 423-6.

23. Goldman M, Thoua Y, Dhaene M, Toussaint C. Necrotising glomerulonefritis associated with cholesterol microemboli. BMJ 1985; 209: 205-6.

24. Peat DS, Mathieson PW: Cholesterol emboli may mimic systemic vasculitis. BMJ 1996; 313: 546-7.

25. Lye WC, Cheah JS, Sinniah R. Renal cholesterol embolic disease. Case report and review of the literature. Am J Nephrol 1993; 13: 489-493.

26. Gillerot G, Sempoux C, Pirson Y, Devuyst O. Wich type of dialysis in patients with cholesterol crystal embolism? Nephrol Dial Transplant 2002; 17: 156-158.

27. Scoble J. Is nihilism in the treatment of atheroembolic disease at an end? Am J Kidney Dis 1999; 5: 975-976.

28. Fabbian F, Catalano C, Lambertini D, Bordin V, Di Landro D. A possible role of corticosteroids in cholesterol crystal embolization. Nephron 1999; 83: 189-190.

29. Boero R, Pignataro A, Rollino C, Quarello F. Do corticosteroids improve survival in acute renal failure due to cholesterol atheroembolism? Nephrol Dial Transplant 2000; 15: 441.

30. Graziani G, Santostasi S, Angelini C, Badalamenti S. Corticosteroids in Cholesterol Emboli Syndrome. Nephron 2001; 87: 371-373.

31. Díaz C, Escuín F, Jiménez C, Picazo ML, Gil F, Costero O, Sanz-Guajardo A. Fracaso agudo por embolismo de colesterol en injerto renal de donante cadáver. ¿Pronóstico más favorable que en riñones nativos? Nefrología 2002; 22: 282-286. 